\title{
Linking modern and ancient technology - the structure of the meeting
}

\author{
R.D. Ekers ${ }^{1}$ \\ CSIRO Astronomy \& Space Science \\ Vimiera \& Pembroke Roads, Marsfield, NSW 2121 \\ Australia \\ E-mail: Ron.Ekers@csiro.au
}

\begin{abstract}
:
More than a hundred years ago an extraordinary mechanism was found by sponge divers at the bottom of the sea near the island of Antikythera in Greece. This Antikythera Mechanism is an ancient computer from about 100BC which uses bronze gears to make astronomical calculations based on cycles of the Solar System. Now, more than 2000 years after the device was lost at sea, scientists have used the latest techniques in X-ray computed tomography and reflectance imaging to understand its intricate workings.

About every 5 years in the village of Kerastari in the ancient region of Arcadia in Greece experts in radio astronomy from all around the world meet to discuss their latest scientific discoveries and astronomical techniques. In June 2012 we held a workshop linking modern and ancient astronomical technology through the Antikythera theme.

We explored the evolution of astrometry and computing from ancient Greece to the present, compared the technologies used to unravel the secrets of the Antikythera Mechanism with the imaging tools of modern astronomy and as we pursued our vision of an exciting scientific future with telescopes such as the Square Kilometre Array we could reflect on why the Antikythera technology was lost for more than a thousand years and whether this could happen again.
\end{abstract}

From Antikythera to the Square Kilometre Array: Lessons from the Ancients, Kerastari, Greece

12-15 June 2012

\footnotetext{
$1 \quad$ Speaker
} 


\section{Introduction to Kerastari workshop}

The Antikythera Mechanism was discovered in a ship wreck off the island of Antikythera in 1900, it was initially assumed to be some sort of astrolabe but this was immediately controversial because it seemed too complex to have been built before the assumed date of the shipwreck in 50 - 80 BC. After initial analysis by Svoronos, Rados, Redrasis and the classical philologist Albert Rehm little more was done until 1953 when De Solla Price recognized the deep significance of the existence of such ancient technology [1]. In 1971 De Solla Price initiated a project to get gamma- and x-radiographs (Oakridge) of the mechanism. In the De Solla Price analysis some of the details may have been wrong but he understood the importance of discovering such advanced technology from circa $80 \mathrm{BC}$. He raised the questions of the climate needed to develop science and technology and asked why the technology disappeared.

I first learned about the Antikythera Mechanism because of my interest in Derek de Solla Price's research in the sociology of scientific discoveries [2]. The vision for this meeting really stems from reading Jo Marchant's book "Decoding the Heavens" [3]. What interested me greatly was the interplay of technologies that were used to decode the mechanism, as well as the historical development of the ideas and culture that produced it. By chance the same technologies of X-ray tomography and surface photometry, and of course the astrometry, are fundamental to aspects of my own field of radio astronomy. So that set me thinking that this would be an incredible opportunity for multidisciplinary cross fertilization.

At a meeting of the Kythera society of Sydney in 2009 there was a talk on the Antikythera machine and exhibition of the first model by Allan Bromley, a computer scientist at the University of Sydney, and this lead to the idea of holding a meeting in Tasso's village of Kerastari, which is only a few hundred km from the site of the Antikythera shipwreck.

In 2005 Tony Freeth and Mike Edmunds from the UK with John Seiradakis, Yanis Bitsakis and Agamemnon Tselikas from Greece obtained funding for "The Research Project" [4] and this research will dominate the discussions in this meeting.

\section{Multidisciplinary discussions}

We have collected a cross section of the key people who have been involved in either the astronomy, the history, or the technology needed to decode the Antikythera Mechanism and we have some of the world experts in radio astronomy and associated technologies. This is neither an expert meeting on the Antikythera Mechanism itself, nor a meeting on modern radio astronomy, but rather an opportunity to generate ideas by exploring the links between developments of modern and ancient astronomical technology. Quite a few of us are also involved in the international project to build a Square Kilometre Array so it's interesting to think of this as a contemporary parallel to the Antikythera Mechanism, and hence the meeting title.

We are a very diverse group so keep this in mind when making presentations. It is always hard to communicate across discipline boundaries so avoid use of jargon. Remember that the 
value of crossing boundaries will more than compensate for the need to go slowly. I believe we may succeed because the links between the topics are so rich.

\section{Unifying themes}

\subsection{Astronomy}

i. Positional astronomy provides an unbroken link from ancient to modern astrometry.

ii. The development of science and technology underpins all modern astronomy. Here we concentrate on radio astronomy developments as is appropriate for the Kerastari meetings.

iii. The proposal to build the Square Kilometre Array (SKA) introduces a visionary modern astronomy project to compare with projects from the past.

\subsection{Imaging}

i. X-ray tomography and radio synthesis imaging

$\mathrm{X}$-ray tomography is directly linked to the imaging procedures used in synthesis radio telescopes. Until the 60's X-ray and radio imaging were unrelated fields, then there was a spectacular period of interaction, involving people like Ron Bracewell at Stanford, which lasted for about a decade when it was realised the mathematical foundations are identical and that the cross fertilization resulting from sharing ideas could be very productive. Only occasionally since then we have had multidisciplinary meetings on indirect imaging and in the case of astronomy this has usually been with the medical imaging profession.

ii. Surface imaging

The Hewlett-Packard laboratory team have applied Polynomial Texture Mapping (PTM) to examine the surface inscriptions on the Antikythera Mechanism. This technique has some relationship to the photogrametry technique for measuring complex three dimensional shapes of objects such as radio astronomy antennas. In general, there are links between many aspects of astronomical imaging and image processing in art conservation and archaeology.

\subsection{Innovation in computing, science and engineering}

i. The Antikythera Mechanism is sometimes described as the world's first computer. The SKA is considered to be one of the first "software telescopes".

ii. Examination of drivers for technology and innovation suggests parallels between ancient and modern times.

\subsection{Society}

i. Why do societies support scientific development?

ii. How are large projects managed - then and now?

iii. Is the SKA - an example of the future generation of an Antikythera Machine? 


\section{References}

[1] D. J. de Solla Price, Gears from the Greeks: the Antikythera Mechanism, a Calendar Computer from ca. 80 BC, Trans. Am. Phil. Soc., Vol. 64, part 7, 1974.

[2] D. J. de Solla Price, Little science, big science, Columbia University Press, 1963.

[3] J. Marchant, Decoding the Heavens: A 2,000-Year-Old Computer--and the Century-Long Search to Discover Its Secrets, Arrow Books Ltd., 2008.

[4] T. Freeth et al, Decoding the ancient Greek astronomical calculator known as the Antikythera Mechanism, Nature 444, pp587-591, 2006. 